Research Article

\title{
Study on the Seismic Performance of Small-Diameter Bolts Reinforced in Grottoes
}

\author{
Ningbo Peng $\mathbb{D}^{1,2}$, Bo Sun $\mathbb{D D}^{3,4}$ Jizhong Huang $\mathbb{D}^{1},{ }^{1}$ Yun Dong $\mathbb{D}^{2}{ }^{2}$ and Ye Zhu $\mathbb{D}^{2}$ \\ ${ }^{1}$ Institute of Applied Mathematics and Mechanics, Shanghai Key Laboratory of Mechanics in Energy Engineering, \\ School of Mechanics and Engineering Science, Institute for Conservation of Cultural Heritage, Shanghai University, \\ Shanghai 200444, China \\ ${ }^{2}$ Faculty of Architecture and Civil Engineering, Huaiyin Institute of Technology, Huai'an 223001, China \\ ${ }^{3}$ Northwest Research Institute Co. Ltd. of China Railway Engineering Corporation, Lanzhou 730000, China \\ ${ }^{4}$ Key Laboratory of Mechanics on Disaster and Environment in Western China, The Ministry of Education of China, \\ School of Civil Engineering and Mechanics, Lanzhou University, Lanzhou 730000, China
}

Correspondence should be addressed to Ye Zhu; zhuye1986@hyit.edu.cn

Received 2 February 2021; Accepted 1 March 2021; Published 11 March 2021

Academic Editor: Alessio Cascardi

Copyright (C) 2021 Ningbo Peng et al. This is an open access article distributed under the Creative Commons Attribution License, which permits unrestricted use, distribution, and reproduction in any medium, provided the original work is properly cited.

\begin{abstract}
The Yungang Grottoes, a World Heritage Site in Datong, consist of 252 caves that are noted for their collection of 5th- and 6thcentury Buddhist grotto sculptures and reliefs. Various diseases have appeared in the grottoes under the general influence of natural and artificial factors. Bolt support is a commonly employed method for grotto reinforcement and has been widely applied in many projects. Small-diameter bolts have also been used in the reinforcement projects at the Yungang Grottoes, but the corresponding effect on the seismic performance of grottoes is still unclear. In this paper, a dynamic analysis via the numerical modelling of an ear grotto of the 19th grotto in Yungang is established, and the rock displacement, acceleration, and bolt axial force responses under a seismic wave are analyzed. The results show that the seismic dynamic responses of grottoes are greatly affected by the cliff structure. The displacement and acceleration responses of the cliff body vary greatly within the abrupt transition of the cliff structure. Based on this variation, the seismic capacity of small-diameter bolts in the vertical direction is greater than that in the horizontal direction. The axial force of a bolt is small at both ends of the bolt, large in the middle of the bolt, small on the top of a cliff, and large at the bottom of the cliff. Although the axial force is small, the upper rock mass of the grotto has a tendency to undergo relative movement compared with the outer rock mass. The results also indicate that based on the structural defects in the vertical direction of the cliff body caused by grotto excavation, the inclined angle of the bolt should be increased as far as possible or vertical support should be adopted to enhance the stability of the rock mass at the top of the grotto.
\end{abstract}

\section{Introduction}

A grotto cliff body is a special kind of artificial rock slope that is usually dug on the edge of a cliff body. This digging causes cliff bodies to become steep inclined slopes with large inclined angles that are nearly vertical to the ground. As investigated, grottoes have irregular shapes and a variety of statues [1]. After years of weathering and human destruction, cracks have appeared in rock masses of grottoes, which tend to produce more diseases [2-4].

Investigation shows that the collapse and destruction of numerous grottoes are related to earthquakes, especially in areas with strong seismic activity. Historically, grotto temples, as permanent cultural relics, are always at the risk of earthquakes. As recorded, nearly $70 \%$ of grotto temples in China were destroyed because of their location in highintensity and earthquake-prone areas [1]. For example, the Tiantishan Grottoes in Wuwei, Gansu Province, China, were seriously damaged in the Wuwei Earthquake (1927), which caused the collapse and irreversible destruction of numerous grottoes, while the nearby unexcavated mountain did not experience major damage, and no large cracks appeared. The overall situation is still being mitigated. This case shows that the existence of the grottoes has a substantial impact on the 
stability of the mountain structure, and the destruction of the grottoes by the earthquake is disastrous. Therefore, an investigation of the seismic safety of grottoes is critical to reduce the impact of earthquake disasters.

Anchoring can strengthen and reinforce the weak or broken rock and soil mass with materials that have relatively large stiffness; it substantially improves the self-stability of the rock and soil mass, which stabilizes the engineering structure $[5,6]$. Bolts have been widely applied in rock and soil mass support engineering, including grottoes [7]. Many research achievements on bolt seismic reinforcement have been made, but most of them were developed by analyzing the slope, underground chamber, and tunnel reinforcement engineering. These previous studies cannot fully illustrate the seismic mechanism of grottoes because these rock heritages are more unstable than the slope and more open than the underground chamber; they also have more dimensions and characteristics than tunnels. Logically, the seismic analysis of grottoes is quite different from that of slopes, underground chambers, and tunnels.

In the reinforcement and protection project of the Yungang Grottoes, small full-length bonded bolts have been installed in many spots. Practically, small-diameter bolts can improve the integrity of fractured rock mass and cause few disturbances to the cultural relic body. However, an evaluation system or method for the aseismic effect of a small anchor on the grotto rock is lacking. To explore the aseismic effect on the cliff bodies of grottoes, the ear grotto of the 19th grotto in Yungang was adopted as a research target, in which a numerical model of grottoes and anchoring were established. By this study, the seismic effect and principle of reinforcing rock grottoes with small bolts are analyzed by numerical calculation under dynamic action.

\section{Rock Slope Analysis Model}

2.1. Grotto Overview. The Yungang Grottoes in Datong city, Shanxi Province, represent the outstanding achievement of Buddhist art in China in the 5th and 6th century, with 252 caves and 51,000 statues. The Yungang Grottoes, Mogao Grottoes in Dunhuang, and Longmen Grottoes in Luoyang are known as China's three largest ancient grottoes; they are famous as world cultural heritages. Under the action of longterm natural forces, the surrounding rock of these grottoes has suffered numerous serious geological diseases [8-10].

The total protection area of the Yungang Grottoes is approximately $3.6 \mathrm{~km}^{2}$, with the Shili River in the south and low hills in the north. The geographical terrain of the grottoes is slightly undulating, with a maximum height difference of approximately $55 \mathrm{~m}$. The geomorphologic types of the grottoes can be generally divided into two forms: a high platform structure with denudation in the low hills at the top of the Yungang Grottoes and valley erosion accumulation on the terrace of the Shili River [11]. The grotto rock mass has moderate softness and hardness, which is suitable for carving, but has poor resistance to weathering. The composition of the 19th grotto consists of grayish-white medium sandstone on the top of the grotto with a distribution between 4 and $5 \mathrm{~m}$; medium sand and fine sandstone with mudstone and sandy mudstone in the interlayer with a distribution between 5 and $10 \mathrm{~m}$; and light brown and grayish-white medium sand and fine sandstone $10 \mathrm{~m}$ above the grotto $[12,13]$. The geological section of the grotto cliff body is shown in Figure 1. To obtain the calculation parameters of the rock mass that occurs in the grottoes, four types of rocks were taken from the same stratum of the mountain near the grottoes (Figure 2(a)), and the rocks were cut into samples for test (Figure 2(b)). The physical and mechanical parameters of the rock mass were obtained by experiments (Figures 2(c) and 2(d)). The parameters of the rock mass that occurs in the grottoes are shown in Table 1.

The 19th grotto of the Yungang Grottoes is one of the most representative grottoes at the research site. The main cave is oval in shape, with a vault roof, a door, and clear windows. The height of the seated Buddha in the main cave is $16.8 \mathrm{~m}$, and it is the second highest statue in the Yungang Grottoes. One ear grotto is cut approximately $5 \mathrm{~m}$ from the ground to the east and west of the grotto, in which a sitting statue with a height of $8 \mathrm{~m}$ is carved. The research object of this paper is the west ear grottoes and their cliff bodies, as shown in Figure 3(a). After an earthquake, the fore wall of the 20th grotto and the west ear grotto of the 19th grotto collapsed. Part of the cave wall between the ear grotto and the main grotto is relatively thin, and there is a broken hole on the cave wall, as shown in Figure 3(b). The thinnest part is less than $10 \mathrm{~cm}$; the cause of this damage is unknown.

2.2. Calculation Model and Boundary Conditions. In previous research, the time-history analysis of the seismic dynamics of the grottoes' cliff bodies is relatively rare. Grottoes are usually reduced to regular caves, while the statues in grottoes are large in size and irregular in shape, which affect the stress distribution of their cliff bodies. The geometries of the grottoes may have greater influence on the dynamic response under dynamic action. Therefore, the shapes of the statues in the grottoes should not be disregarded in the dynamic response analysis of the grottoes' cliff bodies, and the morphologies of the grottoes should be reflected in the model for better observation or modelling. The three-dimensional model of the grottoes' cliff bodies is established, and the three-dimensional point cloud of the facade and the inner main grotto walls of the grottoes' cliff bodies are obtained. The finite element software ANSYS is employed for auxiliary modelling, and the model is imported into FLAC3D for calculation. The built model is shown in Figure 4(a). The total height of the model is $29.25 \mathrm{~m}$; the eastwest ( $X$-axis direction) width is $12.12 \mathrm{~m}$; the north-south ( $Y$ axis direction) length is $20 \mathrm{~m}$; and the model has 48,029 nodes and 255,463 units. Because the cracks in the grottoes' cliff bodies have been treated by grouting, the influence of cracks is not considered in this model.

2.3. Anchorage Design and Calculation Parameters. Small bolts are applied to reinforce the upper rock mass of the ear grottoes, as shown in Figure 4(b). A full-length bonded bolt is adopted for support: the length of the bolt $l=7 \mathrm{~m}$; transverse and vertical spacing $D=2 \mathrm{~m}$; angle $=10^{\circ}$; bolt 


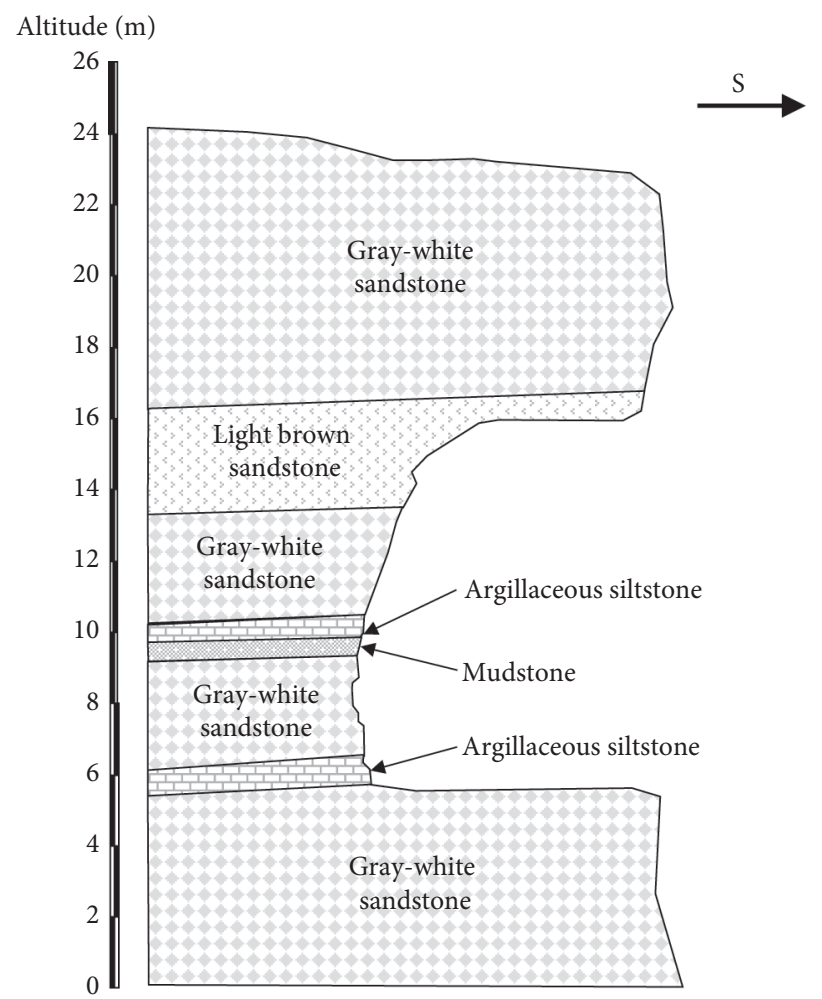

Figure 1: Typical geological section profile of the study area.

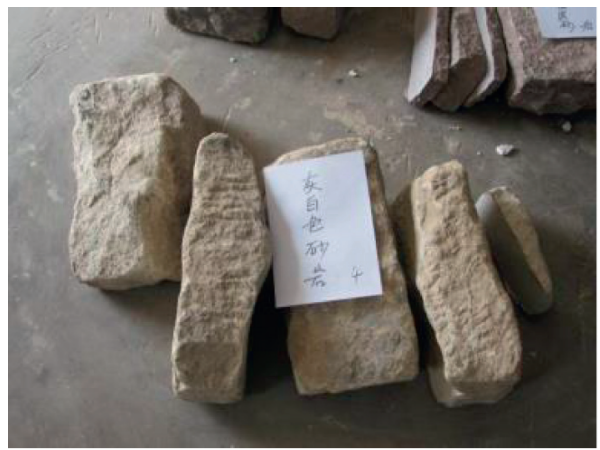

(a)

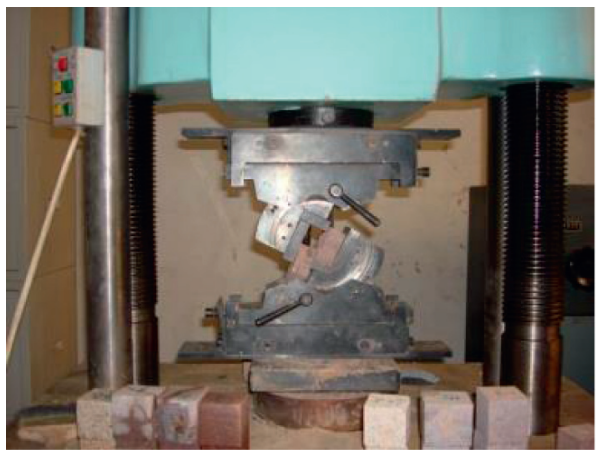

(c)

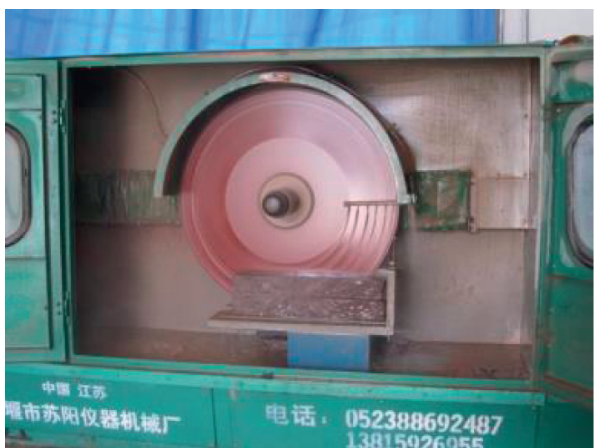

(b)

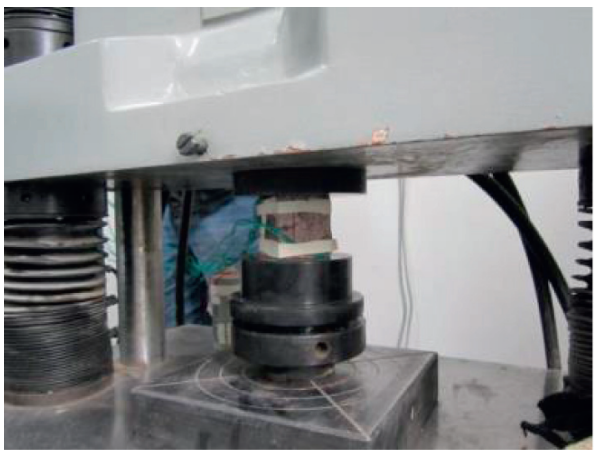

(d)

FIGURE 2: Rock mechanics experiment: (a) primary sample, (b) sample cutting, (c) rock shear test, and (d) uniaxial compression experiment.

diameter $r=12 \mathrm{~mm}$; and design load is $40 \mathrm{kN}$. From the left boundary of the model, a row of bolts was installed with an interval of $2 \mathrm{~m}, 5$ vertical rows, 4 bolts in each row, and a total of 20 small bolts. For the convenience of analysis, 5 rows of bolts were numbered as groups X1 to X 5 from left to right along the $x$-axis. From low to high along the elevation 
TABLe 1: Physical and mechanical parameters of the rock mass in the grottoes.

\begin{tabular}{lcccccc}
\hline Rock type & $\begin{array}{c}\text { Density } \\
\left(\mathrm{g} \cdot \mathrm{cm}^{-3}\right)\end{array}$ & $\begin{array}{c}\text { Elasticity modulus } \\
(\mathrm{GPa})\end{array}$ & $\begin{array}{c}\text { Poisson's } \\
\text { ratio }\end{array}$ & $\begin{array}{c}\text { Cohesion } \\
(\mathrm{MPa})\end{array}$ & $\begin{array}{c}\text { Internal friction } \\
\text { angle }\left(^{\circ}\right)\end{array}$ & $\begin{array}{c}\text { Tensile strength } \\
(\mathrm{MPa})\end{array}$ \\
\hline $\begin{array}{l}\text { Gray-white } \\
\text { sandstone }\end{array}$ & 2.16 & 27.90 & 0.198 & 1.0 & 30.45 & 5.39 \\
$\begin{array}{l}\text { Light brown } \\
\text { sandstone }\end{array}$ & 2.55 & 23.70 & 0.144 & 0.8 & 32.13 & 1.25 \\
$\begin{array}{l}\text { Argillaceous } \\
\text { siltstone }\end{array}$ & 1.79 & 13.39 & 0.298 & 0.5 & 14.07 & 0.01 \\
Mudstone & 1.71 & 8.29 & 0.280 & 0.3 & 13.13 & 0.02 \\
\hline
\end{tabular}

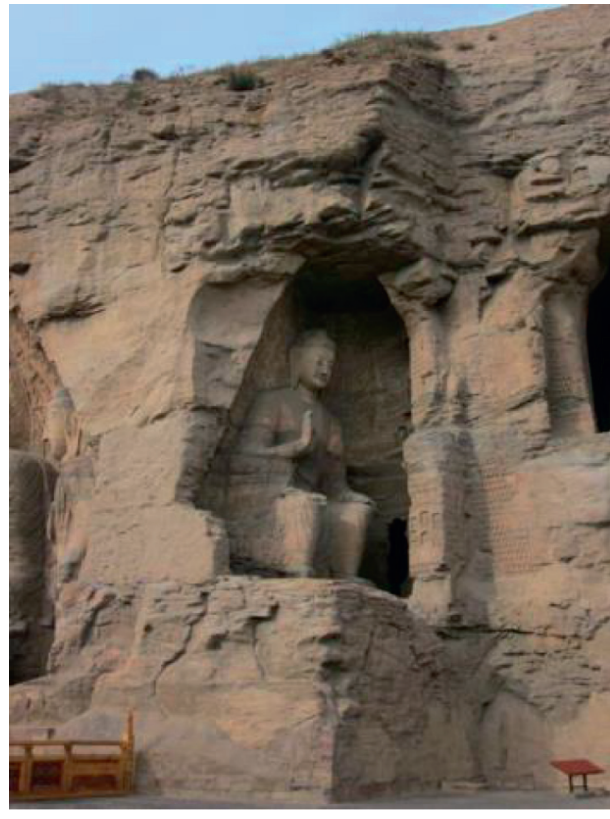

(a)

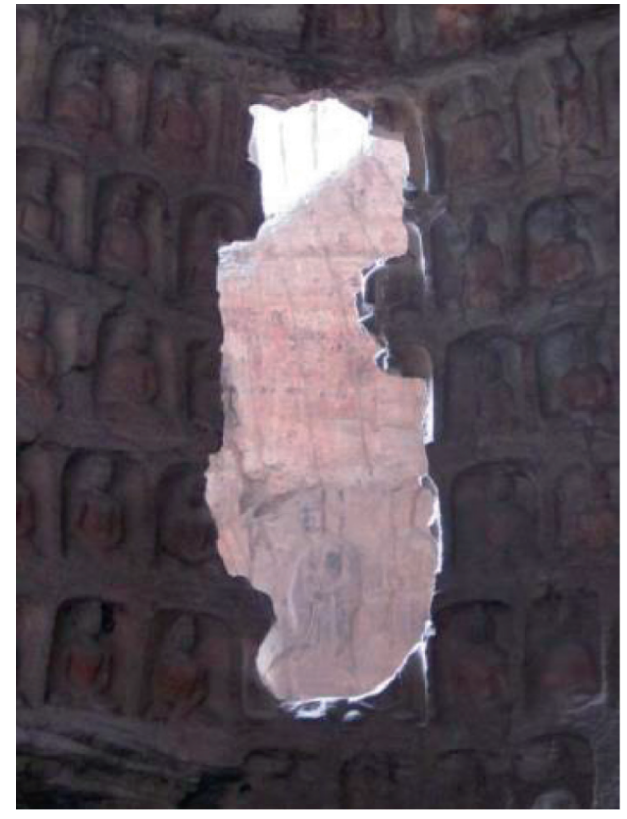

(b)

FIgURE 3: (a) Western ear grotto of the 19th grotto in Yungang. (b) Damage to the thin wall between the ear grotto and the main grotto.

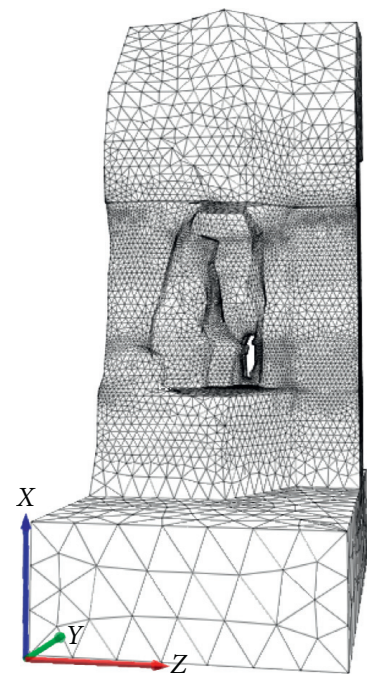

(a)

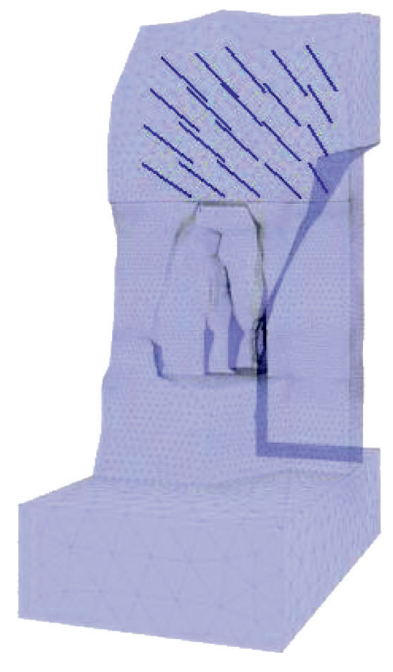

(b)

Figure 4: Numerical model: (a) numerical model of the unanchored grotto and mesh generation; (b) schematic of bolt support design. 
direction, the bolts were numbered as groups $\mathrm{Z1}$ to $\mathrm{Z} 4$. According to the position, the bolts could be numbered as group $\mathrm{XZ}$, in which $\mathrm{X}$ and $\mathrm{Z}$ represent the $x$-direction of the bolts and the $z$-direction of the bolts, respectively.

2.4. Cable Structural Elements. Structural units provided for anchor/cable simulation in FLAC3D include Cable and Pile [14]. The axial stress of the small bolt, which has a small diameter and a weak bending resistance, is considered. Therefore, a cable is chosen to simulate the anchor rod within the research. Each cable structural element is defined by its geometric, material, and grout properties. CableSEL is assumed to be a straight segment of uniform cross-sectional and material properties that is located between two nodal points. An arbitrarily curved structural cable can be modeled as a curvilinear structure that is composed of a collection of cableSELs. CableSEL behaves as an elastic-perfectly plastic material that can yield in tension and compression but cannot resist a bending moment.

Each cableSEL has a unique coordinate system, as shown in Figure 5, to define the average axial cable direction. The cableSEL coordinate system is defined by the locations of its two nodal points, which are labeled 1 and 2 . The cableSEL coordinate system is defined as follows:

(1) The centroidal axis coincides with the $x$-axis

(2) The $x$-axis is directed from node 1 to node 2

(3) The $y$-axis is aligned with the projection of the global $y$ - or $x$-direction (whichever is not parallel with the local $x$-axis) onto the cross-sectional plane

The two active degrees of freedom of the cable finite element are shown in Figure 5. For each axial displacement shown in the figure, there is a corresponding axial force. The stiffness matrix of the cable finite element includes a single degree of freedom at each node, which represents axial action within a cable structure.

Naturally, the shear behavior of the cable-rock interface is cohesive and frictional. Within this model, the system is conceptualized and represented numerically as a springslider system that is located at the nodal points along the cable axis. In evaluating the axial forces that develop in the reinforcement, displacements are computed at the nodal points along the reinforcement axis, as shown in Figure 6. Out-of-balance forces at each node are computed from the axial force in the reinforcement, and the shear forces contributed via the shear interaction along the grout annulus. Axial displacements are computed by integrating the nodal accelerations using the out-of-balance axial force and a mass that is lumped at each node.

In the calculation of anchoring, the grotto model without anchoring is analyzed, and the boundary conditions of dynamic-static force, monitoring point setting, and input seismic waves remain the same as the original model. Only the bolt element is added to the upper rock mass of the original model. The material parameters of the small bolt in the model are shown in Table 2.

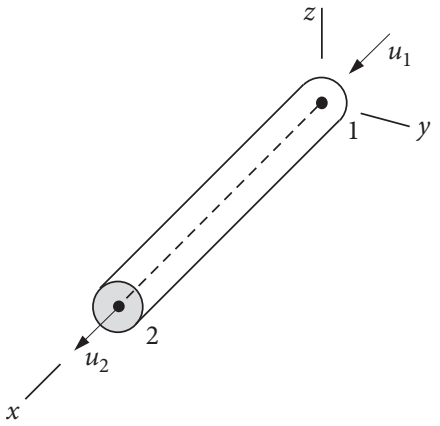

FIgUre 5: CableSEL coordinate system and 2 active degrees of freedom of the cable finite element.

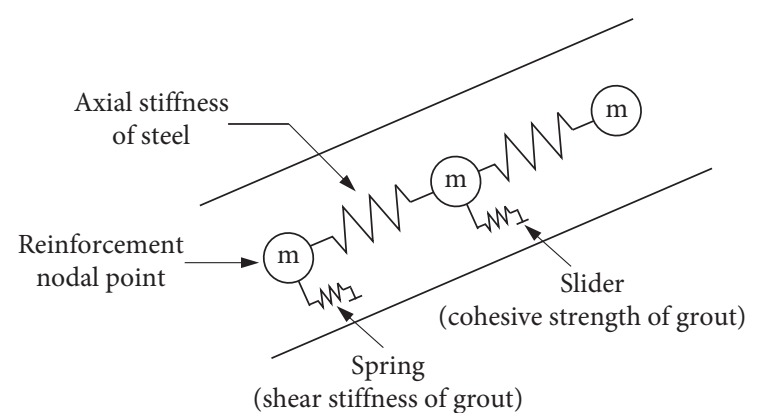

Figure 6: Mechanical representation of fully bonded reinforcement.

2.5. Boundary Conditions and Seismic Waves. Numerical analysis of the seismic response of surface structures, such as dams, requires the discretization of a region of the material adjacent to the foundation. The seismic input is normally represented by plane waves that propagate upward through the underlying material. The boundary conditions at the sides of the model must be accounted for in the free-field motion that exists in the absence of the structure. These boundaries need to be placed at distances that are sufficient for minimizing the wave reflections and achieving free-field conditions. To apply the free-field boundary in FLAC3D (Figure 7), the model should be oriented such that the base is horizontal, its normal is in the direction of the $z$-axis, the sides are vertical, and their normals are in the direction of either the $x$ - or $y$-axis.

The Kobe seismic wave with a maximum positive acceleration of $2 \mathrm{~m} / \mathrm{s}^{2}$ is selected as the input seismic wave in the calculation of the seismic force within the grotto cliff body. The duration of the seismic wave is $18 \mathrm{~s}$, and the preeminent frequency range is $1-3 \mathrm{~Hz}$. The input direction is positive in the $y$-axis. The time-history curve of acceleration is shown in Figure 8.

2.6. Monitoring Stations. To facilitate the subsequent analysis and generate the final calculation results, monitoring points were established before processing the calculation. The distribution of the monitoring points is shown in Figure 9. A total of 38 monitoring points in three groups-E1-E12, M1-M14, and W1-W12-were set up along the 
TABLE 2: Material parameters of the small bolt.

Modulus of elasticity $(\mathrm{GPa})$ Yield load $(\mathrm{kN})$ Bond stiffness $\left(\mathrm{N} / \mathrm{m}^{2}\right)$ Bond strength $(\mathrm{N} / \mathrm{m})$ Anchor bar diameter $(\mathrm{mm})$ Anchor hole

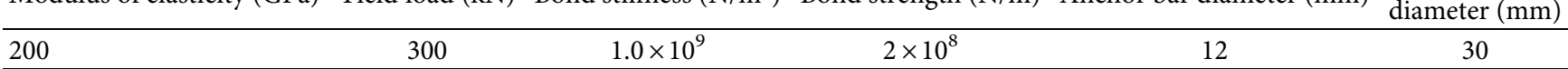

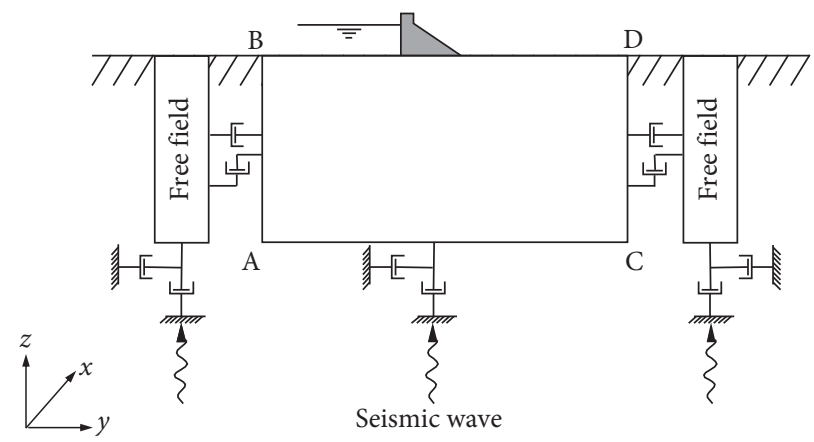

Figure 7: Model for seismic analysis of surface structures and free-field mesh.

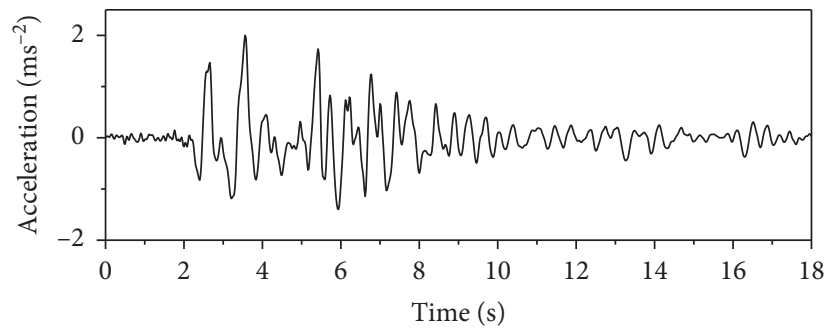

FIGURE 8: Input seismic wave of the model.

elevation at different $x$-coordinates of the model facade, as shown in Figure 9(a). A group of 12 monitoring points were set up along the elevation of the inner wall of the model, namely, the wall of the main grotto, as shown in Figure 9(b).

\section{Analysis Results}

3.1. Displacement Response. To analyze the influence of the small bolt support on the horizontal seismic permanent displacements of grottoes, the $y$-direction relative displacement-time history curve of the small bolt support is compared with the bolt-free support of the grotto cliff body at some monitoring points (consider group M's monitoring points as an example), as shown in Figure 10(a).

On the position of different monitoring points, the final horizontal displacement of the anchored cliff is larger than that of the unanchored cliff, as shown in Figures 10(b)$10(\mathrm{~d})$. For monitoring point M9 (Figure 10(c)), where the difference in the displacement between the two points is relatively large, the final horizontal relative displacement of the unanchored cliff body is $-29.57 \mathrm{~mm}$ and the anchored cliff body is $-29.95 \mathrm{~mm}$. After anchoring, the horizontal relative displacement of the cliff body increases by $0.38 \mathrm{~mm}$, which is approximately $1.29 \%$; this increase can be considered negligible.

Under the support of small bolts, the horizontal displacement of the seismic response of the grotto cliff body increases slightly because of the difference in the seismic wave propagation caused by the improvement in the integrity by anchoring the rock mass. The difference between the anchored rock mass and the unanchored rock mass is almost negligible; the support of small bolts has almost no influence on the $y$-direction displacement response of the grottoes under the action of earthquake. This finding indicates that the small bolts have no clear function in reducing the permanent displacement of the cliff body in the horizontal direction.

Although only the axial force is considered in the calculation process of the cable unit, there is a certain angle between the anchor bolt and the horizontal displacement with a reflection of the effect on the vertical displacement of the cliff body. To analyze the effect of the bolt support on the settlement of grottoes in the $z$-direction, the uneven settlement of the rock inside and outside the top of the model is compared before and after anchoring, as shown in Figure 11. For the majority of the seismic action, the settlement value of the anchored cliff is less than that of the unanchored cliff. The largest difference between the anchored cliff and the unanchored cliff occurs when the seismic action lasts for $7.654 \mathrm{~s}$. The uneven settlement of the unanchored cliff body and anchored cliff body is $12.180 \mathrm{~mm}$ and $9.437 \mathrm{~mm}$, respectively, which is a reduction of $22.52 \%$. At the end of the seismic action, the uneven settlement of the unanchored cliff and anchored cliff was $32.970 \mathrm{~mm}$ and $31.593 \mathrm{~mm}$, which is a 


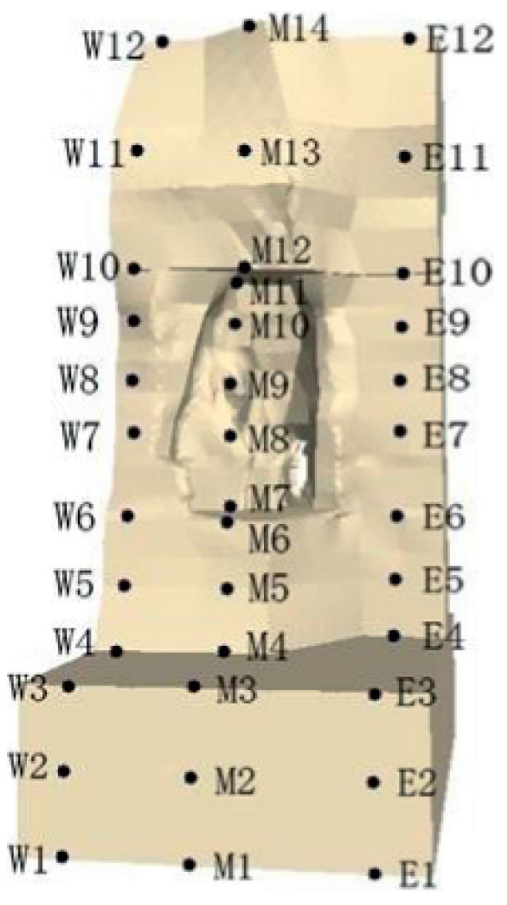

(a)

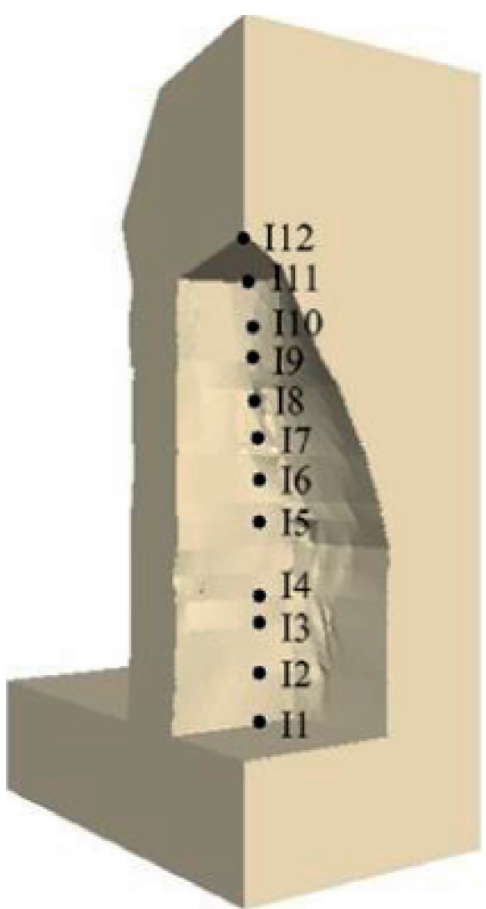

(b)

FIGURE 9: Distribution of monitoring points: (a) a total of 38 monitoring points were set up in groups E, M, and W on the model facade; (b) inner wall monitoring points were set up with a group of 12 monitoring points.

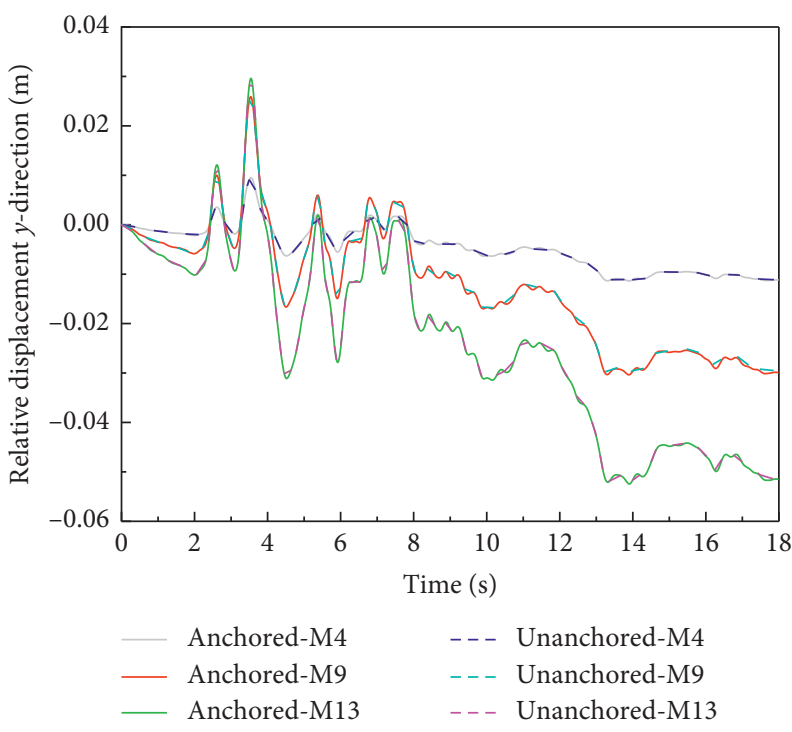

(a)

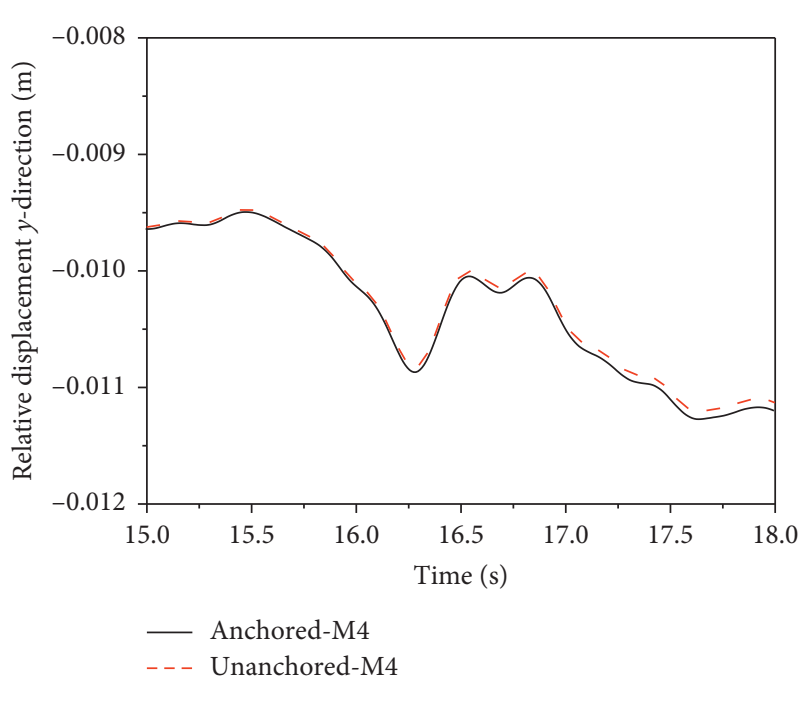

(b)

Figure 10: Continued. 


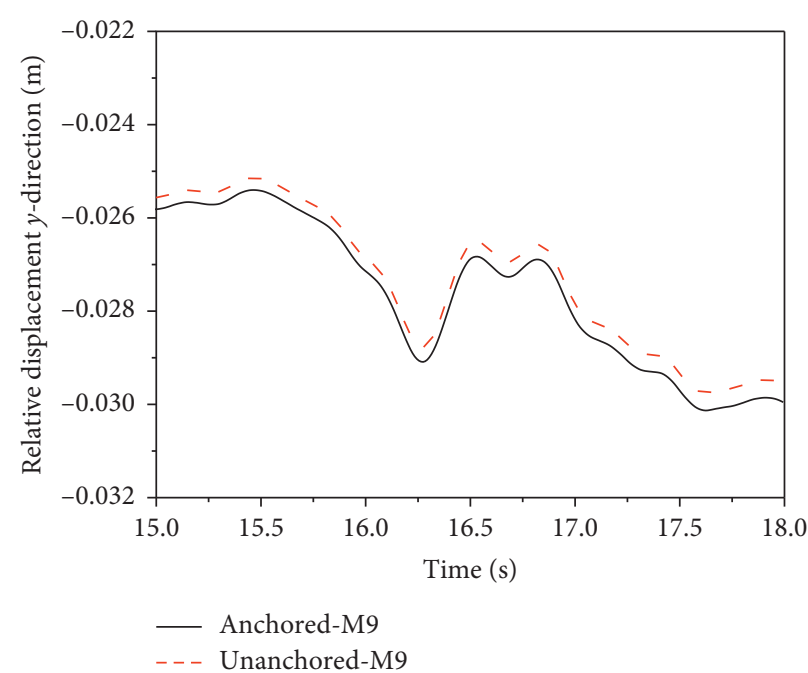

(c)

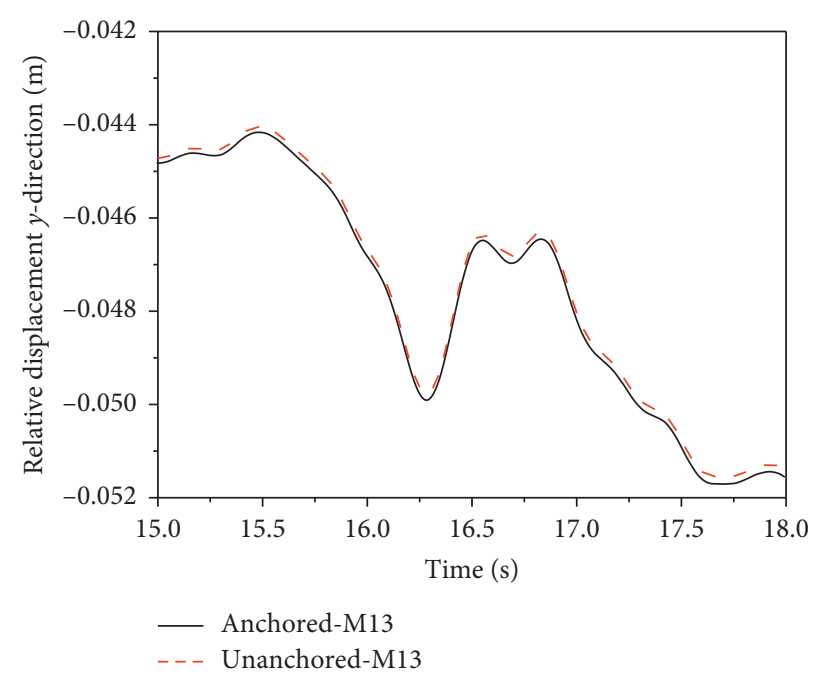

(d)

Figure 10: Relative displacement in the $y$-direction of anchorage grottoes: (a) relative horizontal displacement-time history of some monitoring points with and without anchoring; (b) local magnification of relative horizontal displacement-time history at monitoring point M4 with and without anchoring; (c) local magnification of relative horizontal displacement-time history at monitoring point M9 with and without anchoring; (d) local magnification of relative horizontal displacement-time history at monitoring point M13 with and without anchoring.

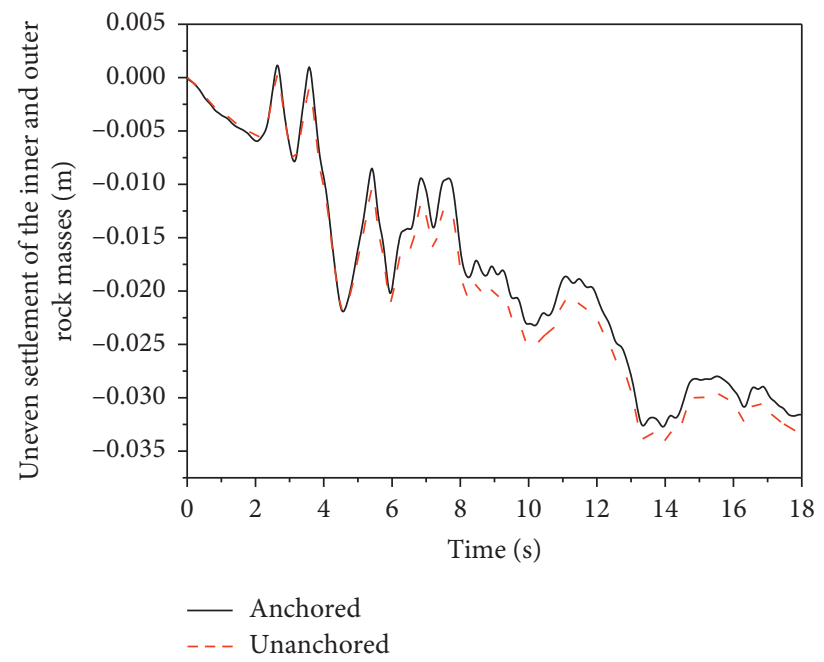

FIGURE 11: Uneven settlement curves of grottoes with and without anchors.

reduction of $1.377 \mathrm{~mm}$ or $4.18 \%$. Therefore, for different moments of earthquake action, the effect of the anchor on the uneven settlement of the cliff body is inconsistent. By comparison, the effect of the anchor on the vertical settlement of the grotto is slightly more significant than that of the horizontal displacement of the grotto. However, the seismic effect of the small bolt is still not ideal, with regard to the reduction in the final displacement, because the seismic effect of the small bolt is not fully realized.

3.2. Acceleration Response. Within the dynamic calculation of the slope, the slope surface usually amplifies the effect of seismic waves. To explore the ground motion response law of the grotto cliff body, the PGA (peak ground acceleration) amplification factor is defined as the ratio between the peak acceleration response of each record point and the peak acceleration response at the bottom of the model. Figure 12 shows the line diagram of the PGA amplification factor, which varies within the elevations of the 4 sets of monitoring points.

The PGA amplification factor of the cliff body shows an initial increasing trend and then a decreasing trend, among which monitoring point M10 was near 130. This trend also presents an order of magnitude that differs from the PGA amplification factor that has been investigated by previous 


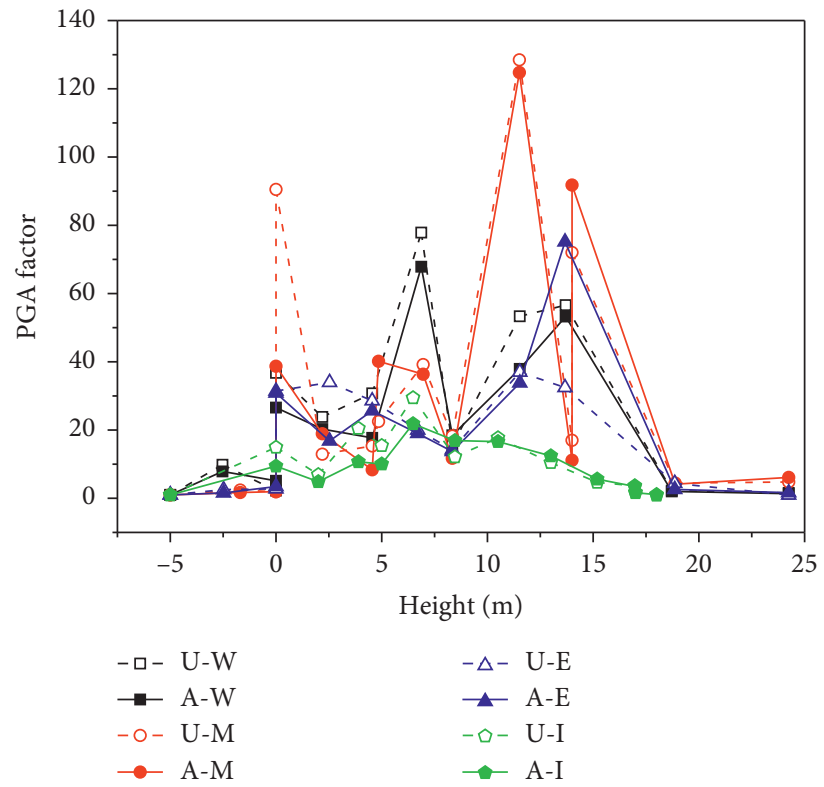

Figure 12: PGA amplification factor at different monitoring points of the anchorage and original cliff body.

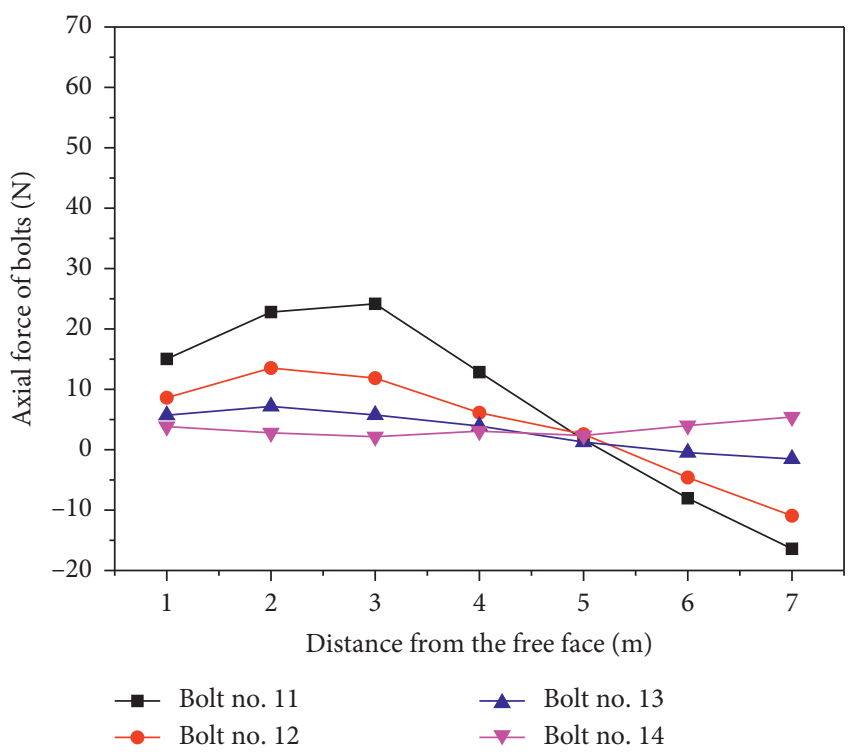

(a)

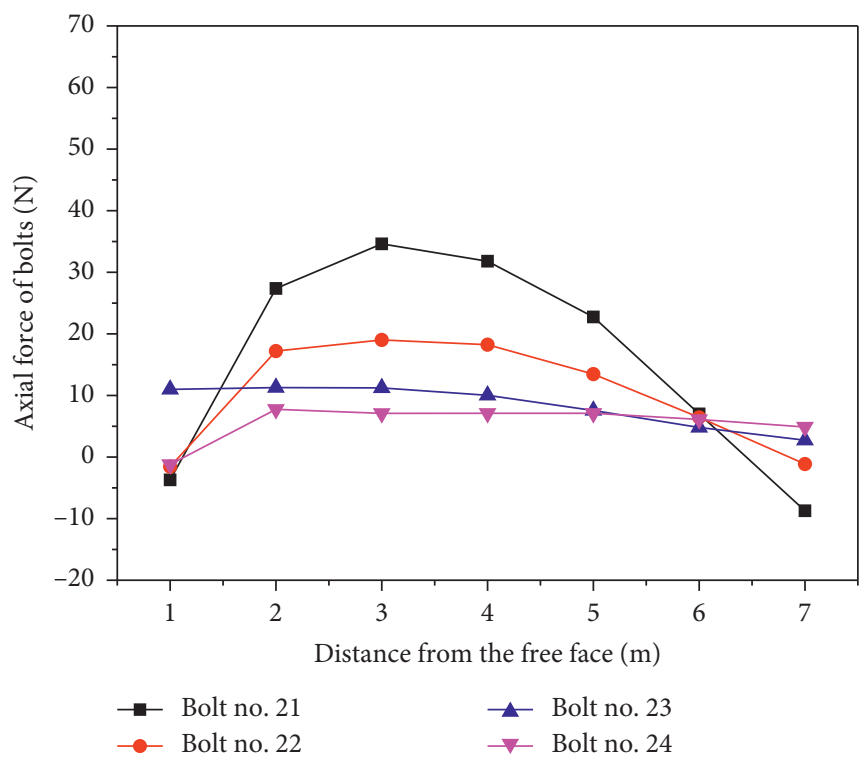

(b)

FIGURE 13: Continued. 


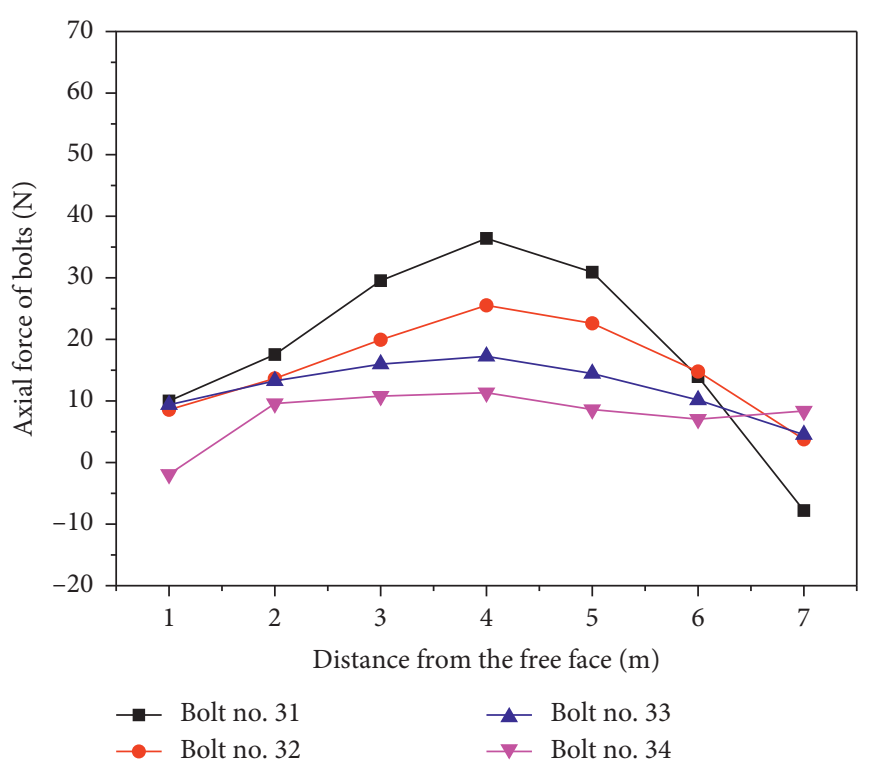

(c)

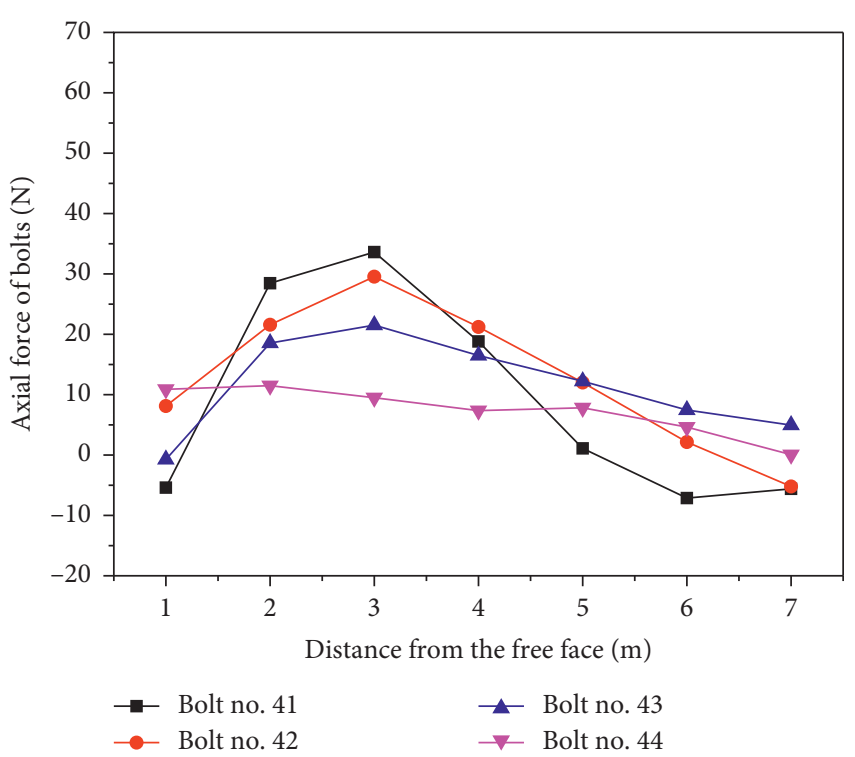

(d)

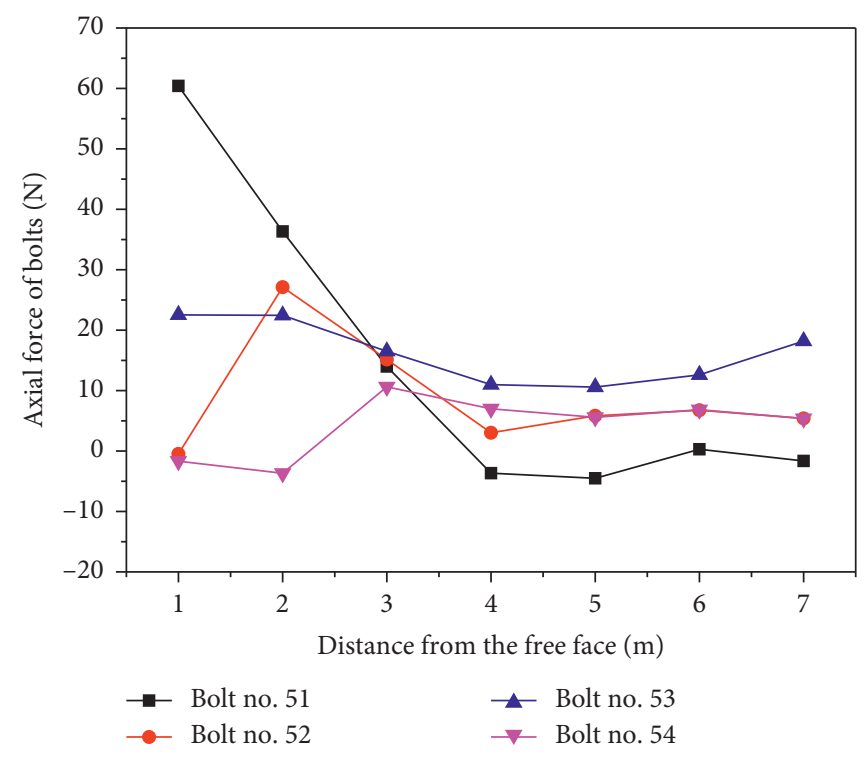

(e)

Figure 13: Bolt axial force distribution.

scholars in the dynamic response of the slope. The PGA amplification factor does not increase along the elevation; it first increases and then decreases and presents a strong fluctuation. Apparently, the PGA amplification factor of the monitoring point in the elevation area where the ear hole is located is relatively large because the digging makes the rock mass of grottoes have a special geometric structure. The rock mass has two groups of irregular facing surfaces, and its geometric characteristics are more complex. During the process of seismic wave propagation, there will be more reflection and superposition between the two groups of surfaces, which is more complicated than the normal engineering slope. Considerably, the significant increase in the PGA amplification factor prompts the installation of monitoring devices to achieve a better illustration of the effect.

It can also be shown in Figure 12 that, at the point of geometric structure mutation, the PGA amplification factor changes significantly even if the elevation remains constant, such as M3 and M4, between M11 and M12. Although they are located at the same height, their PGA amplification coefficient difference is very large. This finding further indicates that the geometry of the rock mass has a considerable influence on its seismic dynamic response. From the top of the grottoes to the top of the model, the PGA amplification factor decreases sharply, which indicates that the seismic wave dissipates more at the location of the rock at the top of the ear grottoes. 
The analysis of the PGA amplification coefficient of the anchor-reinforced cliff body and original cliff body reveals that the PGA amplification coefficient of the anchor-reinforced cliff body and cliff body without anchoring is different but still retains the original cliff body distribution characteristics of the PGA amplification coefficient. The PGA amplification coefficient shows a decreasing trend after the initial increase, and in the elevation range of ear grottoes, the PGA amplification factor is relatively large.

3.3. Axial Force Response of the Bolt. By the analysis of the displacement and acceleration response of the anchorreinforced cliff body, it can be seen that the anchoring effect of the small bolt is not fully exerted, and the axial force response of the bolt is small. The axial force distribution of the bolt is shown in Figure 13. With regard to the overall distribution, most of the bolt axial force is still small at both ends and large in the middle, with a smaller bolt axial force. As reflected by one of the most significant axial forces, the force of the no. 51 bolt (Figure 13(e)) is only $60.42 \mathrm{~N}$, which is substantially less than its design load of $40 \mathrm{kN}$. This small axial force does not fully exhibit its anchoring effect. The analysis confirms that the bolt has no significant effect on the seismic reinforcement. The analysis results of the axial force of the bolts among different groups indicate that the maximum axial force of the bolts in each group decreases, which is consistent with the increase in elevation and is similar to the distribution law of the axial force of the bolts in the homogeneous anchored slope [15].

\section{Conclusion}

As indicated by this analysis, the horizontal displacement of the anchor-reinforced cliff body is increasing rather than decreasing; this trend is also observed in the region without the anchor support. This condition is attributed to the difference in the seismic wave propagation caused by the changes in the material properties of the original cliff body after installation of the anchor support. The change in the material properties of the rock mass causes a difference between the distribution of the PGA amplification factor of the reinforced anchor and the original cliff body. Consequently, the use of small bolts for the reinforcement method has no significant influence on the horizontal displacement responses of the grottoes calculated in this study. However, in the vertical direction, there is a certain angle between the bolt and the horizontal direction that can have a certain role in the vertical direction. Within the whole process of seismic action, the uneven settlement of the anchor is controlled to some extent, but the final results show that the seismic effect of the anchor is not ideal.

According to the existing literature, bolt support mainly changes or improves the structural characteristics of the rock mass. Because the fracture and structural plane characteristics of the grotto rock mass are not considered in this study, the structural characteristics of the upper rock mass of the cliff body are not significantly improved in the horizontal direction even with the support of the bolt. Otherwise, within the vertical direction, ear grotto digging produces a discontinuous rock mass defect. Although the force of the bolt in the vertical direction is not large, it still has a certain controlling effect on the uneven settlement of the upper rock mass and can prevent fracture between the inner rock mass and outer rock mass at the top of the grotto. In addition, as the axial force of the bolt is directly related to the relative displacement of the rock around the bolt, with the relatively small displacement of the rock in the cliff body, the axial force response of the bolt is quite small with no indication of its supporting role.

With this calculation model, this kind of support with small bolts cannot reduce the relative displacement of the upper rock mass of the grotto cliff body in the horizontal direction. However, in the vertical direction, the support has a certain role in controlling the uneven settlement of the upper rock mass. The axial force in the bolt is very small, which also indicates that its supporting effect on the rock mass has not been fully realized.

By observing the structural defects in the vertical direction of the cliff body caused by the grotto excavation, the inclined angle of the bolt should be increased as far as possible or the vertical support should be adopted to enhance the stability of the rock mass at the top of the grotto. The results show that the anchoring effect of the bolt on the slope is mainly reflected in changing or improving the structural characteristics of the rock mass under the action of an earthquake.

\section{Data Availability}

All the relevant data underlying this research are included within this paper.

\section{Conflicts of Interest}

The authors declare that they have no conflicts of interest.

\section{Acknowledgments}

This study was financed by the National Natural Science Foundation of China (no. 51808246), National Key Research and Development Program of China (no. 2019YFC1520500), Research Project of China Railway Academy Co., Ltd. (no. 2020KJ009Z009A2), the Six Talent Peaks Project in Jiangsu Province of China (no. JZ-011), the New Wall Materials and Bulk Cement Support Projects of Jiangsu Province (no. 2017-21), and the Open Fund of Jiangsu Engineering Laboratory of Assembly Technology on Urban and Rural Residence Structure (no. JSZP201901).

\section{References}

[1] F. Gao, Y. C. Shi, K. Wei, and R. D. Qiu, "The influence of anchor bolt supports on seismic responses of strengthening grottoes," World Earthquake Engineering, vol. 22, no. 2, pp. 84-88, 2006, in Chinese.

[2] W. Chen, Z. Q. Guo, J. K. Zhang, and F. He, "Evaluation of long-term stability of Mogao grottoes caves under enhanced 
loading conditions of tourists," Journal of Performance of Constructed Facilities, vol. 32, no. 4, 2018.

[3] C. S. Christoforou, L. G. Salmon, and G. R. Cass, "Deposition of atmospheric particles within the Buddhist cave temples at Yungang, China," Atmospheric Environment, vol. 28, no. 12, pp. 2081-2091, 1994.

[4] R. Z. Liu, B. J. Zhang, H. Zhang, and M. F. Shi, "Deterioration of Yungang grottoes: diagnosis and research," Journal of Cultural Heritage, vol. 12, no. 4, pp. 494-499, 2011, in Chinese.

[5] Z. Huang, Stabilizing of Rock Cavern Roofs by Rock Bolts, Norwegian University of Science and Technology, Trondheim, Norway, 2001.

[6] Y. Shi, R. Qiu, J. Sun, and M. Hu, "Analysis of dynamic response of dangerous rock mass reinforced by prestressed anchor cables under seismic loads," Rock and Soil Mechanics, vol. 32, no. 4, pp. 1157-1162, 2011, in Chinese.

[7] X. Wang, Q. Guo, S. Yang, Q. Pei, and Y. Fan, “The engineering geological problems and conservation of cliff face of Dunhuang Mogao Grottoes, China," Engineering Geology for Society and Territory, vol. 8, pp. 183-187, 2015.

[8] Q.-L. Guo, X.-D. Wang, H.-Y. Zhang, Z.-X. Li, and S.-L. Yang, "Damage and conservation of the high cliff on the northern area of Dunhuang Mogao grottoes, China," Landslides, vol. 6, no. 2, pp. 89-100, 2009.

[9] L. Li, J. Du, H. Liu, R. Chen, and T. Liu, "Dynamic characteristics and seismic responses of painted sculptures of Dunhuang Mogao Grottoes," Journal of Cultural Heritage, vol. 22, pp. 1040-1048, 2016.

[10] Q. X. Liu, Y. H. Duan, and J. Y. Deng, "Geological protection project of the longmen grottoes in Luoyang," Advanced Materials Research, vol. 594-597, pp. 155-160, 2012.

[11] J. Huang, "Study on the geological characteristics of Yungang grottoes," Southeast Culture, vol. 5, pp. 91-93, 2003, in Chinese.

[12] E. Yan and Y. Fang, "Quantitative evaluation on the safety of the column rock masses in Yungang Grotto," Chinese Journal of Rock Mechanics and Engineering, vol. 23, no. s2, pp. 5046-5049, 2004, in Chinese.

[13] X. Yang, T. Peng, G. Li et al., "Study on long term strength of pillar rockmass at Yungang Grottoes," Chinese Journal of Rock Mechanics and Engineering, vol. 28, no. s2, pp. 3402-3408, 2009, in Chinese.

[14] Itasca, FLAC3D Version 4.0 (Fast Lagrangian Analysis of Continua in 3 Dimensions) User's Manual, Itasca Consulting Group Inc, Minneapolis, MN, USA, 2009.

[15] H. Ye, Y. Zheng, X. Lu, and A. Li, "Shaking table test on anchor bars of slope under earthquake," China Civil Engineering Journal, vol. 44, pp. 152-157+176, 2011. 